\title{
Hydraulic modelling of drinking water treatment plant operations
}

\author{
G. I. M. Worm ${ }^{1,2}$, G. A. M. Mesman ${ }^{3}$, K. M. van Schagen ${ }^{4,5}$, K. J. Borger ${ }^{6}$, and L. C. Rietveld ${ }^{2}$ \\ ${ }^{1}$ PWN Water Supply Company North-Holland, P.O. Box 2113, 1990 AC, Velserbroek, The Netherlands \\ ${ }^{2}$ Delft University of Technology, Faculty of Civil Engineering and Geosciences, Department of Water \\ Management, P.O. Box 5048, 2600 GA, Delft, The Netherlands \\ ${ }^{3}$ KWR, Watercycle Research Institute, Groningenhaven 7, 3433 PE, Nieuwegein, The Netherlands \\ ${ }^{4}$ DHV, P.O. Box 1132, 3800 BC, Amersfoort, The Netherlands \\ ${ }^{5}$ Delft Center for Systems and Control, Delft University of Technology, Mekelweg 2, 2628 CD Delft, \\ The Netherlands \\ ${ }^{6}$ Vitens, P.O. Box 1090, 8200 BB, Lelystad, The Netherlands
}

Received: 22 September 2008 - Published in Drink. Water Eng. Sci. Discuss.: 21 October 2008

Revised: 29 April 2009 - Accepted: 27 May 2009 - Published: 10 June 2009

\begin{abstract}
The flow through a unit of a drinking water treatment plant is one of the most important parameters in terms of a unit's effectiveness. In the present paper, a new EPAnet library is presented with the typical hydraulic elements for drinking water treatment processes well abstraction, rapid sand filtration and cascade and tower aeration. Using this treatment step library, a hydraulic model was set up, calibrated and validated for the drinking water treatment plant Harderbroek. With the actual valve position and pump speeds, the flows were calculated through the several treatment steps. A case shows the use of the model to calculate the new setpoints for the current frequency converters of the effluent pumps during a filter backwash.
\end{abstract}

\section{Introduction}

Interventions in the operation of drinking water treatment plants, such as the adjustment of valve positions or pump speeds, will lead to a change in the division of flows through the plant and, thus, in the flow through the individual treatment units. The flow through a unit is one of the most important parameters in terms of the unit's effectiveness (Van Schagen, 2009). Hydraulic model studies are commonly part of the design of a drinking water treatment plant (Hranisavljevic et al., 1999) or part of a performance study of a single treatment step (Gallard et al., 2003; Van Schagen et al., 2006). This work focuses on the effects of operational interventions on a complete drinking water treatment plant. The modelling software EPAnet is used worldwide to design water distribution networks and to optimise its operation, up to a level of full integration with SCADA (supervisory control and data acquisition) systems (Martínez et al., 2007; Fontenot et al., 2003). The current EPAnet library, however, lacks elements that describe the hydraulic properties of drinking water treatment plant units such as aerators and rapid sand filters. In this study the use of EPAnet to build a hydraulic model of a drinking water treatment plant is reported. Drinking water treatment plant Harderbroek, consisting of cascade aeration, rapid sand filtration and tower aeration is modelled and the model is calibrated and validated with historical data. The model provides an opportunity for online control the flows of a drinking water treatment plant. The model can be used to support an operation supervisor during (manual) adjustments of pump speeds or valve positions, to evaluate the actual operation, to monitor online flow measurement devices' performance or to serve as a soft sensor at locations where no flow measurement device is available. The Harderbroek model will be integrated in a drinking water treatment simulator (Worm et al., 2009).

\section{Correspondence to: G. I. M. Worm}

(ignaz.worm@pwn.nl) 


\section{Materials and methods}

\subsection{The basic elements of the model}

From a hydraulic perspective a drinking water treatment plant consists of elements that give resistance to the passing flow (e.g. filters, pipes, distribution works), pumps that increase the total head of the flow and reservoirs with a varying water level. A hydraulic model of a drinking water treatment plant can be schematized as pipes and other resistances, pumps and reservoirs, which are connected with junctions. Junctions are not true physical elements but mark points where two or more pipes or valves are connected. An elevation can be assigned to each junction. The total head in a junction is the elevation added up to the pressure in the junction according

$H=\frac{p}{\rho \cdot g}+z$

Where $H$ is the total head (mwc), $p$ is the pressure $\left(\mathrm{N} / \mathrm{m}^{2}\right)$, $\rho$ is the density of the fluid $\left(\mathrm{kg} / \mathrm{m}^{3}\right), g$ is the acceleration due to gravity $\left(\mathrm{m} / \mathrm{s}^{2}\right)$ and $z$ is the elevation of the junction on a chosen level (m). Pipes should be given a length, diameter and roughness. For all pipes in the model described in this paper, the roughness coefficient $k$ is $0.1 \mathrm{~mm}$. Reservoirs are nodes that represent an infinite external source or sink of water. A reservoir's main input property is its hydraulic head. To model resistances in EPAnet (version 2.00.12) six types of valves are available of which four were used in the treatment plant library. These four will be described here (Rossman, 2000). In the design of a drinking water treatment plant often hydraulic disconnections are added to prevent water from flowing in the opposite direction and to distribute water over lanes. For hydraulic disconnections in EPAnet the pressure sustaining valve (PSV) is used. A pressure sustaining valve (PSV) maintains a set pressure at the upstream point. EPAnet computes in which of three different states the PSV is in: i) partially opened to maintain its pressure setting on its upstream side when the downstream pressure is below this value, ii) fully open if the downstream pressure is above the setting or iii) closed if the pressure on the downstream side exceeds the pressure on the upstream. A pressure breaker valve (PBV) forces a specified pressure loss to occur across the valve. Flow through the valve can be in either direction. PBVs are not true physical devices but can be used to model situations where a particular pressure drop is known to exist. A throttle control valve (TCV) simulates a partially closed valve by adjusting the minor head loss coefficient of the valve. The head loss over a TCV is calculated with

$\Delta H=\xi \frac{v^{2}}{2 \cdot g}$

Where $\xi$ is the minor loss coefficient $(-), v=$ is the velocity through the pipe $(\mathrm{m} / \mathrm{s})$ and $g$ is the gravity constant $\left(\mathrm{m} / \mathrm{s}^{2}\right)$. The relationship between opening degree and minor loss coefficient is often given by the valves' manufacturer. A general purpose valve (GPV) is used to represent a link where the user supplies a special flow - head loss relationship instead of following one of the standard hydraulic formulas, like the one mentioned above. The relationship can be linear or quadratic, as well as custom defined.

\subsection{Treatment step library}

The library with the models for a ground water treatment plant is given in Table 1. It contains models for well abstraction, cascade aeration, rapid sand filtration and tower aeration, which are described in the next sections.

Wells. The water level of a freatic aquifer is modelled with a reservoir. Wells can be equipped with a submerged pump or can be part of a vacuum-gravity system to extract the water from the aquifer. As Thiem proposed (Thiem, 1906), for isolated wells with a constant extracted flow the relation between extracted flow and drawdown can be assumed to be linear. For pumped wells, a second non linear term must be added, leading to the following empirical relation (Rorabaugh, 1953) between drawdown and extracted flow

$\Delta z=\frac{Q}{2 \pi T} \cdot \ln \frac{R}{r_{w}}+k \cdot Q^{n}$

Where $Q$ is the extracted flow $\left(\mathrm{m}^{3} / \mathrm{s}\right), T$ is the soil conductivity $\left(\mathrm{m}^{2} / \mathrm{s}\right), R$ the influence well radius $(\mathrm{m}), r_{w}$ the distance to the well (m), $k$ a coefficient to be determined (-) and $n$ an exponent ranging between 1 and $2(-)$. For any situation where the relation between extracted flow and drawdown is known the well draw-down is modelled with a GPV.

Cascade aeration. The points of interest in a cascade aerator, from a hydraulic perspective, are the level of the upper weir and the water level in the last cascade step, or the collection canal or pipe of the cascade effluent. The setting of the PSV is the level of the crest of the upper cascade, see Fig. 1. The GPV represents the height of the water surface above the upper weir. The GPV flow - head loss relationship of the upper weir is calculated for a sharp-crested weir corrected for contractions on both ends of it (Daugherty et al., 1985), assuming the value for the discharge coefficient $C_{D}$ is 0.62 :

$Q=1.84 \cdot(L-0.1 \cdot n \cdot H) \cdot H^{3 / 2}$

where $Q$ is flow $\left(\mathrm{m}^{3} / \mathrm{s}\right), L$ is the width of the weir $(\mathrm{m}), n$ is the number of end contractions and $H$ is the difference in level between the crest and the water in an undisturbed zone in front of the weir (m).

Rapid sand filtration. The library contains a representation of a rapid sand filter with a fixed supernatant water level during the runtime, using a pump or control valve in the effluent pipe that compensates for the increasing filter bed resistance. The total resistance over the filter is mainly caused by the water inlet, the filter bed, the filter bottom nozzles, the effluent pipe inlet and the pump or control valve. The water inlet can be modelled either with a pipe in the case of a siphon, 
Table 1. Treatment step library.

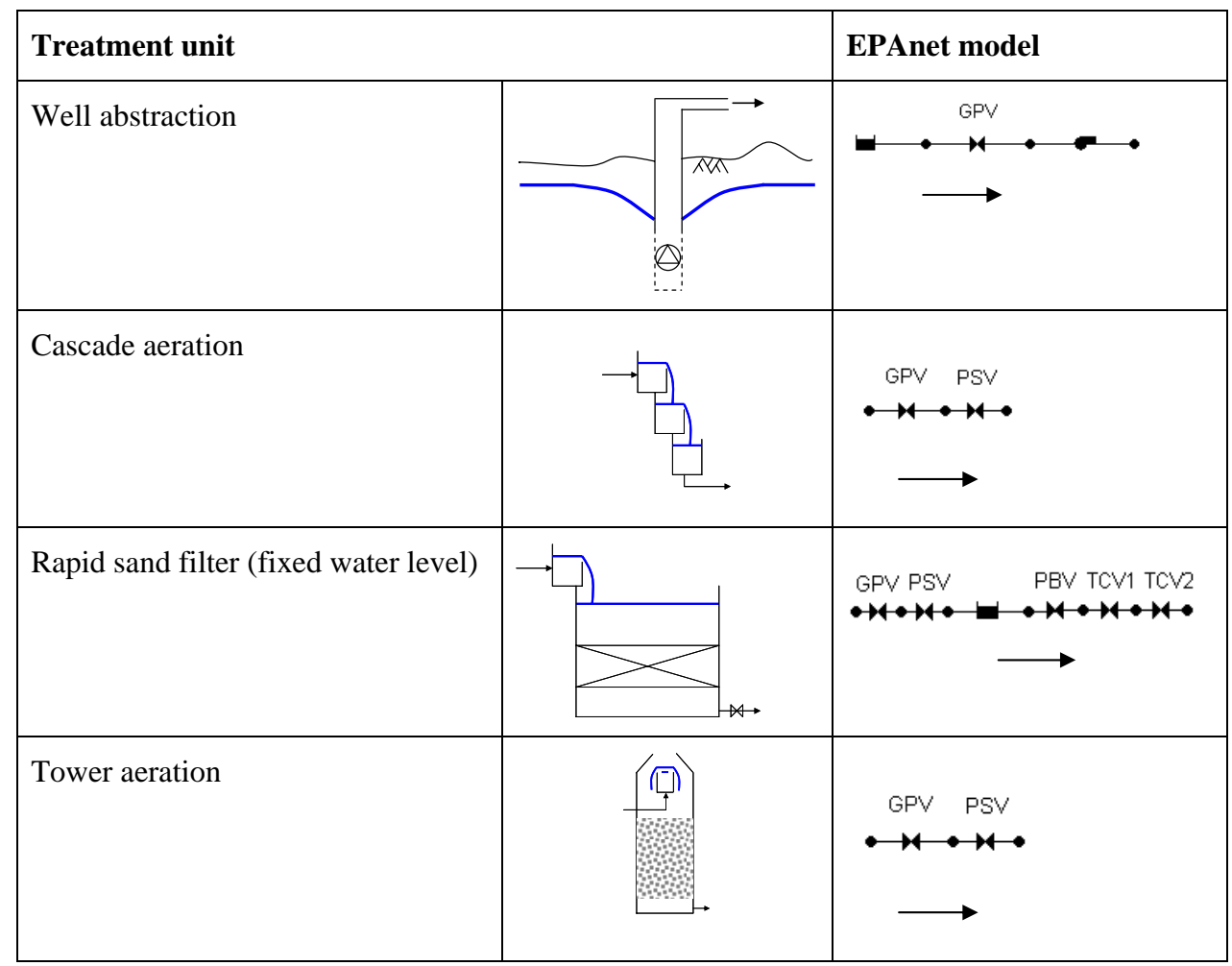

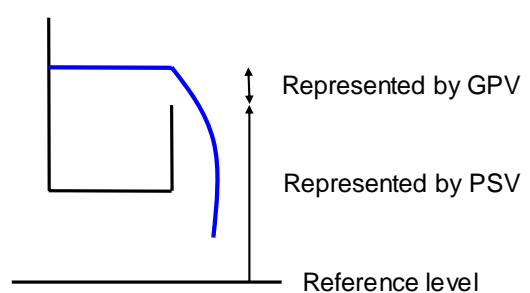

Figure 1. Modelling of the upper weir of a cascade aerator.

with a TCV in the case of a valve, or with a GPV and a PSV in the case of a weir. The pressure drop over the filter bed increases in time as a consequence of clogging or instantly as a consequence of increasing flow and is calculated using a separate water quality model. For a static calculation, the pressure drop as a consequence of clogging is considered to be fixed, and therefore is modelled using a PBV. The resistance of the filter bottom nozzles can often be derived from the specifications of the manufacturer and the number of nozzles. Because of the increasing resistance with increasing flow, the nozzles can be modelled using a TCV. In practice however, the pressure drop over the nozzles during filtration will be negligible. The second TCV simulates the behaviour of the control valve.
Tower aeration. In a tower aerator, water is distributed over a column with packing, through which air is blown. From a hydraulic perspective, the tower aerator is modelled in the same way as the cascade aerator. The height of the weir, plus the flow on top of the crest of the weir, is modelled using a PSV and a GPV.

\subsection{Drinking water treatment plant Harderbroek}

The drinking water treatment plant Harderbroek, owned and operated by Vitens, consists of 16 deep wells, four cascades, eight rapid sand filters and three tower aerators. The treatment schem is shown in Fig. 2. The model was set up using the hydraulic line scheme of the plant, P\&IDs and other technical drawings.

Wells. The wells are grouped in two series of seven. Each well is equipped with a submerged pump, which has been added to the model. In each series, one well is equipped with a speed-controlled pump, the other six are equipped with fixed-speed pumps. The water level inside and outside each well is measured and logged, as is the flow per well. While water level measurements inside each well were available the groundwater level minus actual drawdown was used in the model. In this case the hydraulic head of the reservoir represents the water level in the well in stead of the water level of the aquifer and the GPV was not added. The value 


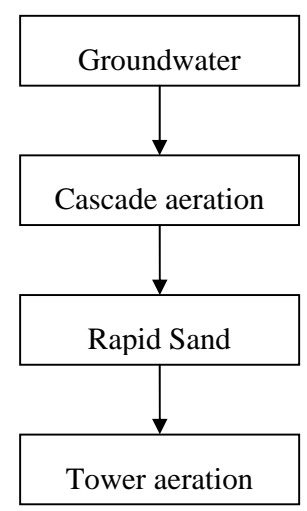

Figure 2. Treatment scheme Harderbroek.

of the water level measurement is the distance between the water level and the sensor at $-13.3 \mathrm{~m}+\mathrm{NAP}$ (Dutch standard level). Based on the pump's characteristic, the water level in the well, pipe resistances and the level of the weir of the upper cascade, the flow per well can be calculated.

Cascades. The top of the weir of each of the four cascades has a level of $4.71 \mathrm{~m}+\mathrm{NAP}$. The relationship between flow and water level is calculated with Eq. (2) above. In a normal operation, three cascades are in operation. After aeration the water from the cascades is collected in the rapid sand filter influent canal.

Rapid sand filters. Each rapid sand filter is fed using an open/close valve and a weir. Each filter has a speedcontrolled pump in the effluent pipe that controls the water level in the filter at a fixed level. This pump replaces the control valve in the library's model. The water level is measured, and so are the pressure drop over the filter and the pressure under the bottom of the filter. The value of the water level measurement equals the distance from the sensor at $3.80 \mathrm{~m}+\mathrm{NAP}$ to the water level. The speed of the pump (expressed as a ratio of the nominal speed [-]) is controlled with a current frequency converter. A pump speed ratio of 0 equals a current frequency of $15 \mathrm{~Hz}$ and a ratio of 1 equals an current frequency of 58. In normal (i.e. average) operation, four rapid sand filters are in operation.

Tower aerators. The counter current tower aerators have their weir at $6.08 \mathrm{~m}+\mathrm{NAP}$. This is the head that the rapid sand filter pumps face upstream. During normal operation, two aerators are in use, and change according to a fixed scheme. Downstream of the aerators, the head in the pipes is determined by the level of the clear water reservoirs.

\subsection{Modelling approach}

To enable integration of the EPAnet model with other systems, iteration within the model was minimized. The model calculated the static hydraulic situation in the water treatment plant for the actual settings (in EPAnet by choosing the total duration of a model-run to be zero). To calculate the resistance in the pipes, the Darcy-Weisbach equation was used. The calibration and validation covered the part from the well to the clear water reservoir. Calibration focused on the minor loss coefficients affecting the distribution of flows over the cascades and the rapid sand filters and on the calculation of the pump speed ratios. Validation focused on the production of the wells at given (inner) well water levels, on the division of flows over the cascades, and on the flows through the rapid sand filters for given pump speeds and water levels. For calibration and validation, nine datasets from the full-scale plant were used within the period 28 June to 23 July 2008.

Calibration. From the randomly picked dataset on 28 June at $10: 30 \mathrm{~h}$, the following input for the model was selected: well water level, the operation of the well pumps ("on" if flow exceeds zero), the operation of the cascades ("on" if flow exceeds zero), the operation of the rapid sand filters ("on" if flow exceeds zero), the water level in the rapid sand filters, the speed of the rapid sand filters' effluent pumps, the operation of the tower aerators and the estimated levels in the clear water reservoirs. Since the speeds of the two speed controlled well pumps lacked in the dataset, the speeds of these pumps were set manually so that the yield of the well in the model equalled the yield in the historical data. After these iterations the model results for validation were captured. The model results of the following parameters were compared with the historical data: flow per well (not the wells containing the two speed-controlled pumps), flow per cascade, influent per filter and effluent per filter.

Validation. For the validation, the same input and output parameters were used as for the calibration. Four validation experiments were carried out: one for the flows from the wells, one for the flows over the cascade aerators, one for the influent and one for the effluent of the rapid sand filters. The experiment consisted of the comparison of the calculated and historical data. For each experiment two moments were selected with a minimum flow, two with an average flow, two with a maximum flow and two during the backwash of a rapid sand filter. Moments with minimum flow occurred on 7 July at 23:30 h and 21 July at 19:30 h; moments with an average flow were on $8 \mathrm{July}, 10: 30 \mathrm{~h}$ and $17 \mathrm{July}, 10: 30 \mathrm{~h}$; with a maximum flow were on 30 June, 15:30 h and $1 \mathrm{July}, 15: 30 \mathrm{~h}$; and with the situation during backwash of a rapid sand filter were on 4 July, 17:30 $\mathrm{h}$ and 23 July, 11:30 $\mathrm{h}$.

\section{Results and discussion}

The model represents a ground water treatment plant with a normal production of $1000 \mathrm{~m}^{3} / \mathrm{h}$. The model contains presentations of the 16 wells, each with a submerged pump, the 4 cascade aerators, the 8 rapid sand filters, each with a pump in the effluent pipe of the filter and the 3 tower aerators. The model contains 344 pipes, 528 junctions and 207 valves. 


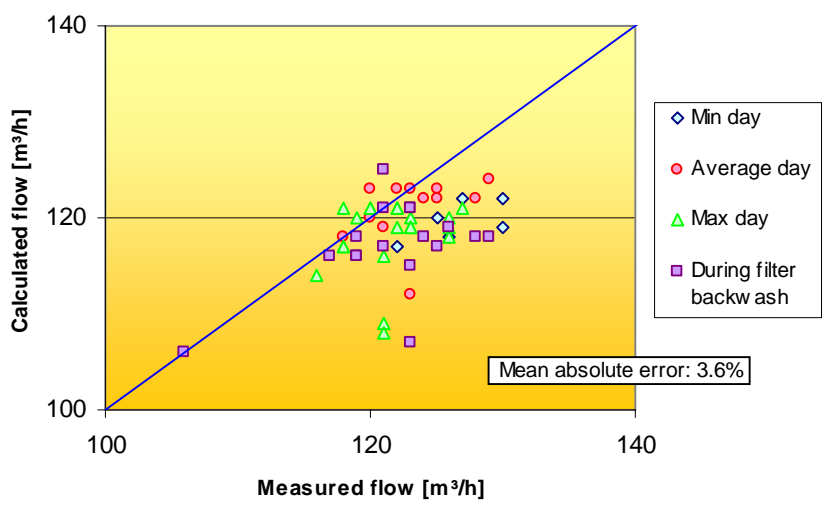

Figure 3. Validation results of flows from wells.

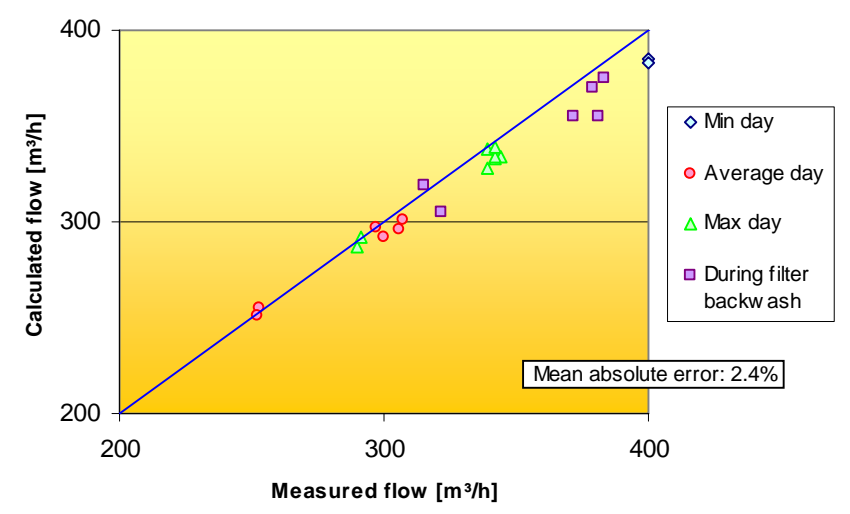

Figure 4. Validation of flows in cascade aerators.

Calibration. Pipe roughness coefficient $k$ was kept constant during calibration. Loss coefficients were adjusted in the way that modelled data met the measured data more closely. The effluent flow of the rapid sand filters appeared to be consistently greater than the measured flow. The factors in the current frequency converter calculation were adjusted: a pump speed of 0 equals a current frequency of $13 \mathrm{~Hz}$ in stead of $15 \mathrm{~Hz}$ and a pump speed ratio of 1 equals a current frequency of $56 \mathrm{~Hz}$ in stead of $58 \mathrm{~Hz}$.

Validation. For the wells, 55 data points were collected. The model shows good results compared to the historical data, Fig. 3. The average of the absolute errors is $3.6 \%$. For the cascade aerators, 22 data points were used. The model results approach historical data as well, see Fig. 4. The average of the absolute errors is $2.4 \%$. For the influent of the rapid sand filters, the average of the absolute errors is $4.4 \%$, based on 34 data points, seen in Fig. 5. When the pressure drop measurements over the filter beds of filters 5, 6 and 8 appeared to be unrealistically small, the pressure drop was estimated by subtracting the pressure measured in the effluent pipe from $26 \mathrm{kPa}$, that being the average pressure of a nonoperating filter. For the rapid sand filters' effluent, the average of the absolute errors is $2.8 \%$, based on 30 data points,

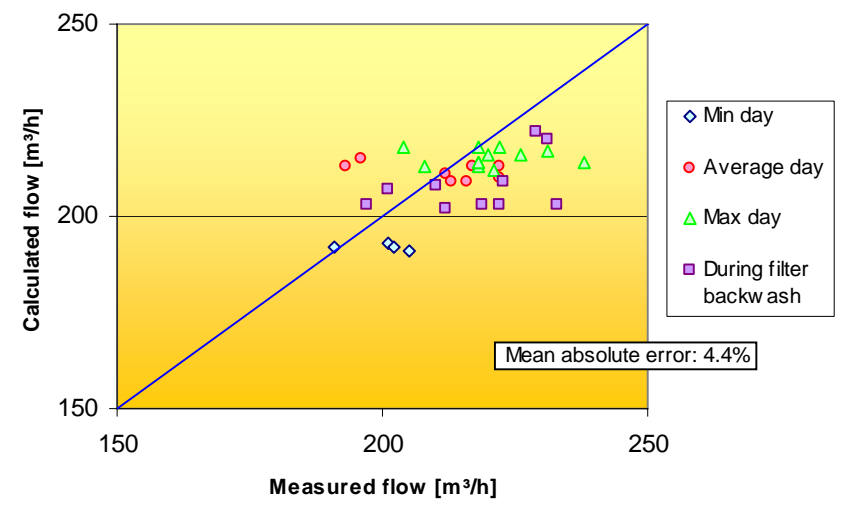

Figure 5. Validation of influent flows rapid sand filters.

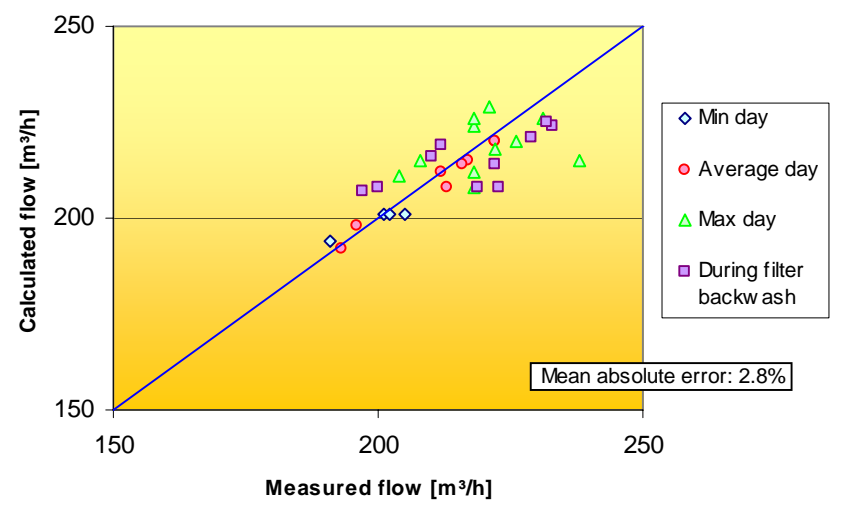

Figure 6. Validation of effluent flows rapid sand filters.

see Fig. 6. In four cases, most probably as a consequence of acceleration during start-up, pump speeds were more than $20 \%$ below the low value of the normal range. In these cases, the pump speeds were replaced by the average speed of the pump and the results were excluded for the validation. The flow over the tower aerators was not measured. For all validation results, it should be noted that any possible inaccuracy of the measuring equipment was not taken into account.

\section{Case: backwash of a rapid sand filter}

During a filter backwash the flows increase over the remaining filters as a consequence of which the filter bed resistances increase too. The model is used to determine the setting of the current frequency converters of the effluent pumps of the remaining filters. The current frequency determines the pump speed.

For a flow of $1015 \mathrm{~m}^{3} / \mathrm{h}$, five rapid sand filters are in operation with a $203 \mathrm{~m}^{3} / \mathrm{h}$ flow each. During backwashing of rapid sand filter 6 , the total flow is divided over the four remaining filters. Small difference in flow between the filters can be seen: filter 2 gets $251 \mathrm{~m}^{3} / \mathrm{h}$, filter 8 gets $256 \mathrm{~m}^{3} / \mathrm{h}$. Since the filter bed resistance is related linearly to the flow (De Moel 
Table 2. Case results: pump speeds ratios (of the nominal pump speed) and current frequencies before and during back wash of sand filter 6.

\begin{tabular}{ccccc}
\hline Filter & \multicolumn{2}{c}{ Before backwash } & \multicolumn{2}{c}{ During backwash } \\
\hline & Pump speed ratio [-] & Current frequency [Hz] & Pump speed ratio & Current frequency [Hz] \\
\hline 1 & 0.94 & 53.4 & 1.12 & 61.2 \\
2 & 0.93 & 53.0 & 1.11 & 60.7 \\
5 & 0.93 & 53.0 & 1.12 & 61.2 \\
6 & 0.93 & 53.0 & 0 & n.a. \\
8 & 0.93 & 53.0 & 1.13 & 61.6 \\
\hline
\end{tabular}

et al., 2006), the $25 \%$ flow increase over each filter yields each filter bed resistance increases with $25 \%$ too. With the increased flows and bed resistances and some iterations, the new pump speed ratios are calculated with the model. With the relation between pump speed and current frequency as mentioned above, the new current frequency for each pump is calculated, see Table 2 . The operation supervisor can apply these setpoints to the frequency converters when he wants to keep the supernatant water level of the rapid sand filters on the same level during filter backwash. This case shows how the model can be helpful for an operation supervisor to calculate the settings to control the pumps in the rapid sand filter effluent pipes.

\section{Conclusions}

Modelling software EPAnet can be used to model the hydraulic behaviour of drinking water treatment plants by using the library described in this paper. With the model the effects of interventions in operation on the division of flows over the plant's lanes or units can be calculated. The library contains models for a well, a cascade aerator, a rapid sand filter and a tower aerator, formed by a series of the basic EPAnet elements valves, reservoirs, junctions and pipes. A model was set up for drinking water treatment Harderbroek and calibrated and validated with historical full-scale plant data. The model can be used to support operation supervisors in daily operation. The model will be part of a drinking water simulator for proactive operation and training.

Acknowledgements. The authors thank Henk Bosma of Vitens for his help in data acquisition for validation. The "Waterspot" project started in February 2007, will end in November 2009, and is a cooperation with PWN, Waternet, Vitens, Duinwaterbedrijf Zuid-Holland, UReason, DHV, ABB, Delft University of Technology and Kiwa Water Research. This research was co funded by SenterNovem, agency of the Dutch Ministry of Economic Affairs with an Innowator subsidy.

Edited by: J. Verberk

\section{References}

Daugherty, R. L., Franzini, J. B., and Finnemore, E. J.: Fluid mechanics with engineering applications, 8 Ed., McGraw-Hill Book Company, New York, 598 pp., 1985.

De Moel, P. J., Verberk, J. Q. J. C., and Van Dijk, J. C.: Drinking water : principles and practices, World Scientific Publishing Co. Pte. Ltd., Delft, 413 pp., 2006.

Fontenot, E., Ingeduld, P., and Wood, D.: Real Time Analysis of Water Supply and Water Distribution Systems, World Water and Environmental Resources Congress, Philadelphia, Pennsylvania, 443-452, 2003.

Gallard, H., Von Gunten, U., and Kaiser, H. P.: Prediction of the disinfection and oxidation efficiency of full-scale ozone reactors, J. Water Supply Res. T., 52, 277-290, 2003.

Hranisavljevic, D., Mazounie, P., MacLean, G. W., and Cox, R. J.: Cooperation between private enterprise and applied research - Prospect Water Filtration Plant, Water Supply, 17, 425-430, 1999.

Martínez, F., Hernández, V., Alonso, J. M., Rao, Z., and Alvisi, S.: Optimizing the operation of the Valencia water-distribution network, J. Hydroinform., 9, 65-78, 2007.

Rorabaugh, M. I.: Graphical and theoretical analysis of stepdrawdown test of artesian well, American Society of Civil Engineers, Ann Arbor, Mich., 1953.

Rossman, L. A.: EPANET 2 Users manual, National Risk Management Research Laboratory, Water supply and water resources division, Cincinnati, 138, 2000.

Thiem, G.: Hydrologische methoden, J. M. Gebhardt's Verlag, Leipzig, 1906.

Van Schagen, K. M., Babuska, R., Rietveld, L. C., and Baars, E. T.: Optimal flow distribution over multiple parallel pellet reactors: A model-based approach, Water Sci. Technol., 53, 493-501, 2006.

Van Schagen, K. M.: Model-based control of drinking-water treatment plants, PhD thesis, Delft, 2009.

Worm, G. I. M., Van der Helm, A. W. C., Lapikas, T., Van Schagen, K. M., and Rietveld, L. C.: Integration of models, data management, interfaces, and training and decision support in a drinking water treatment plant simulator, Environ. Modell. Softw., accepted for publication, 2009. 